\title{
Board Certification in Pediatric Dentistry: Once it Represented the Pursuit of Excellence
}

\author{
John E Nathan ${ }^{1-6 *}$ DDS, MDS, FAAPD, MASDC \\ ${ }^{1}$ Adjunct Professor, Department of Pediatric Dentistry, University of Alabama, Birmingham and Case Western Reserve University, USA \\ ${ }^{2}$ Clinical Associate Professor, Department of Otolaryngology and Dentistry, Northwestern University, Feinberg School of Medicine, USA \\ ${ }^{3}$ Diplomate, American Board of Pediatric Dentistry, USA \\ ${ }^{4}$ Fellow, American Academy of Pediatric Dentistry, USA
}

${ }^{5}$ Fellow and Master, American Society of Dentistry for Children, USA

${ }^{6}$ In private practice limited to pediatric dentistry, USA

*Corresponding author: John E Nathan, DDS, MDS, FAAPD, MASDC, Adjunct Professor, Department of Pediatric Dentistry, University of Alabama, Birmingham and Case Western Reserve University, Clinical Associate Professor, Department of Otolaryngology and Dentistry, Northwestern University, Feinberg School of Medicine, Diplomate, American Board of Pediatric Dentistry, Fellow, American Academy of Pediatric Dentistry, Fellow and Master, American Society of Dentistry for Children, In private practice limited to pediatric dentistry, USA

\begin{abstract}
The scope and changes to the examination format for Board Certification by the American Board of Pediatric Dentistry has successfully achieved the goal of enticing and attracting significantly greater numbers of AAPD members to pursue and complete the board process. Unlike earlier times (prior to 2002), when only 15\% of its membership secured Diplomate status, changes in the examination format that reduced the process from four extensive and demanding components to two much abbreviated elements, approximately $80 \%$ of its membership has now pursued and achieved certification. For better or worse, standards have been radically modified from that of elite and prestigious to that of minimally acceptable. Movement in the direction of making the examination process more attractive and simplified has become the rule rather than the exception. The bar has been sufficiently lowered by the nature of the examination changes. In so doing the demand and requirements for pursuit of knowledge has diminished. A priority for expanded membership at a significant cost has overshadowed the pursuit of excellence among the masses. The old school of thought envisioned inherent motivations to continually seek excellence and the most comprehensive level of knowledge. Its opposing school determined that inclusion of those unwilling or unable to master the highest level of competency remained deserving of recognition. Among the dilemma created by this shift in priorities is that once one fractionates the degree of difficulty and challenge to a lesser level, it is unlikely the direction can be reversed any time soon.
\end{abstract}

\section{Introduction}

What it once meant to achieve Diplomate status from the American Board of Pediatric Dentistry appears no longer to be what it once represented. In the early 1980's approximately 15\% of the membership of the American Academy of Pediatric Dentistry sought and achieved this prestigious credential. Those achieving this lofty accolade were generally recognized as among the best and brightest in the field. Aspirations to secure Diplomate status were essentially limited to those with the broadest range of knowledge and commitment to excellence and lifelong learning.
For whatever reasons, it became apparent that the rigorous nature of the existing four part format remained a deterrent for many to pursue Diplomate status. It became clear that examination format changes represented the only course of action for the board to increase its membership. During the following decade or so, changes in the perception of what this credential represented underwent an evolutionary change foreign to what purists think of today as a movement in an upward direction. The compass appears to have deviated in the direction of lowering rather than 
a raising of standards, let alone the pursuit of excellence. This was not limited to pediatric dentistry; rather instead, most medical and dental disciplines acknowledged that there was a greater need to secure and encourage larger numbers of their constituents to demonstrate this credential. In so doing, practicality and economics dictated that the ultimate attraction to accomplish this objective was that certification examinations must be abbreviated, and the bar lowered as needed to include rather than limit greater numbers from achieving certification.

Amidst the rationale for change included the fact that hospital bylaws in the late 1980's began adopting the position that if a specialty had an American Board, that its members would need to demonstrate certification at the time of re-appointment. Significance for pediatric dentists was their ability to make use of hospital operating rooms for general anesthesia. For those unwilling to pursue board certification, the use of itinerant anesthesiologists and free-standing surgical centers was and has become an alternative, without consequences of not having achieved board certification. Nevertheless, until the format of the examination was reduced, from a rigorous four part process to a significantly abbreviated two part format, the gross numbers electing to pursue certification was not increased. To demonstrate that a significant portion of its members pursued Diplomate status, the ABPD was compelled to re-examine its criteria for certification. Perceptions of its existing degree of difficulty, time commitment, and ultimate outcome gave rise to recognition that without a substantial revision in format, increase in those pursuing certifications was unlikely. Revisions of the four part examination to an abbreviated two part exam became inevitable in not only pediatric dentistry but included orthodontics and other specialty areas. In doing so, a complete renovation in thinking took hold in that redefinition of standards was needed to permit increasing numbers to qualify as diplomates within their field. The pursuit of excellence gave way to what constituted for some, mediocrity or minimally adequate. Frank recognition that requirements for current day certification markedly differ from those of before goes largely unacknowledged. Historically, the original format comprised an all-day written examination, generated from a defined list of 200 carefully selected and identified classical as well as contemporary articles spanning all areas of pediatric dentistry. Each multiple choice question was referenced to a specific article. Successful completion of this preliminary component granted eligibility to participate in Section 2 (a no holds barred one hour oral examination before two examiners). Section 3 consisted of the submission of 5-6 specific types of cases treated which included detailed and meticulous write-ups, pre- and post-treatment radiographic evaluation, with follow-up care to demonstrate diagnostic, technical skill and clinician judgment. Upon successful completion, applicants qualified for part 4 an all-day clinical site visit in which any aspect of clinical care and practice was evaluated by two examiners, in one's clinical or academic setting.
Effective in 2001, the four section format was reduced to two sections. Part one consisted of a half-day multiple choice written examination, based on an unidentified list of texts and papers from the literature. Satisfactory completion permitted candidates to pursue section 2 which served as a $11 / 2 \mathrm{hr}$ clinical simulation on a small range, in essence replacing sections 2,3 and 4 . During this period, for one year, till 2002, candidates had the option of choosing between the four part format and the abbreviated two part formats. In recognition that the new format constituted an abbreviated revision, concerns emerged with respect to a potential need for development of a Re-Certification component within a ten year span. Over the next decade, consistent with the concept of Re-Certification developed by the ABPD and other disciplines, a protocol with intent to demonstrate a propensity for Diplomates to continue to manifest a course of lifelong learning resulted in the formulation of a 50 question, open-book multiple choice recertification examination mandatory for those having passed the two part format (granted limited Diplomate status every ten years). $80 \%$ correct responses were needed to pass. Unlimited Diplomates (those having completed the more rigorous four part format) today remain exempt or voluntarily excused from recertification unless they chose to participate in any capacity related to examination development or appointment to the board.

\section{Differences in Qualification for those having completed the more comprehensive examination format}

At present, there is no distinction amongst which format was undertaken and Diplomate status rewarded other than the subtlety of limited vs unlimited status and a requirement for Re-Certification for those with limited status. Questions remain whether the fifty question open book multiple choice re-certification exam constitutes a valid comparison between examination formats. An argument might be made that individuals who subjected themselves to the four part format possess a sufficient commitment to lifelong learning and keep abreast of new and changing literature. If this premise is accepted, would it not be appropriate to provide distinction in Diplomate status in some recognizable way. The AAPD has reviewed and re-assessed the granting of Fellowship status in the last few years and as such, might consider re-assigning qualification acknowledgment of differential levels of Diplomate status.

For those having completed the four part format, the pursuit of excellence applies; from the perspective of the shortened format, adequacy is implied. The two accolades are distinctly different from one another yet grant equal recognition which appear unfair if not inappropriate. In the course of the past decade, this author has interviewed numerous board certified candidates in search of both academic and private practice positions. Query of knowledge and competency across many fundamental areas of pediatric dentistry including pediatric medicine, recognition and management of medical emergencies, simplified as well as sophisticated 
sedation techniques, thorough understanding of transitional dentition analysis, cephalometric and orthodontic diagnosis and mechanics, and clinical experience can often at best be described as weak. It should not be surprising that a significantly abbreviated certification process does not possess the latitude to include some or all of these areas in detail. Helpful to analysis of the merit of the different schools of thought along with respective format changes, the ABPD might seek to secure a survey of its the membership who have experienced both sides of the examination format. Similarly, it may wish to explore perceptions of the value and validation of the current Re-Certification requirement that impacts on candidates who have completed the contemporary reduced examination format. A survey to accomplish such is currently underway by this author.

\section{Summary}

The decision of the American Board to reduce the directive from the pursuit of excellence to one of lessor proficiency and competency has been both diligently conceived and scrutinized. Keeping pace with other disciplines who similarly saw need to increase their certification membership, the ABPD made the decision that Board Certification should not have as its mission the quest for excellence, that a hint in its direction is enough to inspire its membership. In all fairness, this change was made and influenced by both internal and external pressures. Among external influences was societal pressure to increase the numbers of health care providers who were acknowledged to possess a certain degree of competency through their respective disciplines. Low numbers of board certified members within a given field implied that the majority fell seriously short of what the public should expect. Increased demand for competency as mandated by certification served as a logical outcome for medical societies to re-examine their criteria and expectations for certification. The notion of distinction and elite knowledge while once selected as optimal, has been replaced by an expectation of what constituted a minimally acceptable level of competency achievable by most of its providers. These distinctions comprise a dynamic process the ABPD is strongly committed toward improvement. While critical analysis is in general both easy and prevalent, the task for which the ABPD undertakes is by no means an easy one.

\section{Disclaimer}

The opinions expressed in this editorial are those of the author and in no way be construed to represent or reflect the views of the ABPD or AAPD.
To Submit Your Article Click Here: Submit Article

DOI: $10.32474 /$ IPDOAJ.2019.02.000146

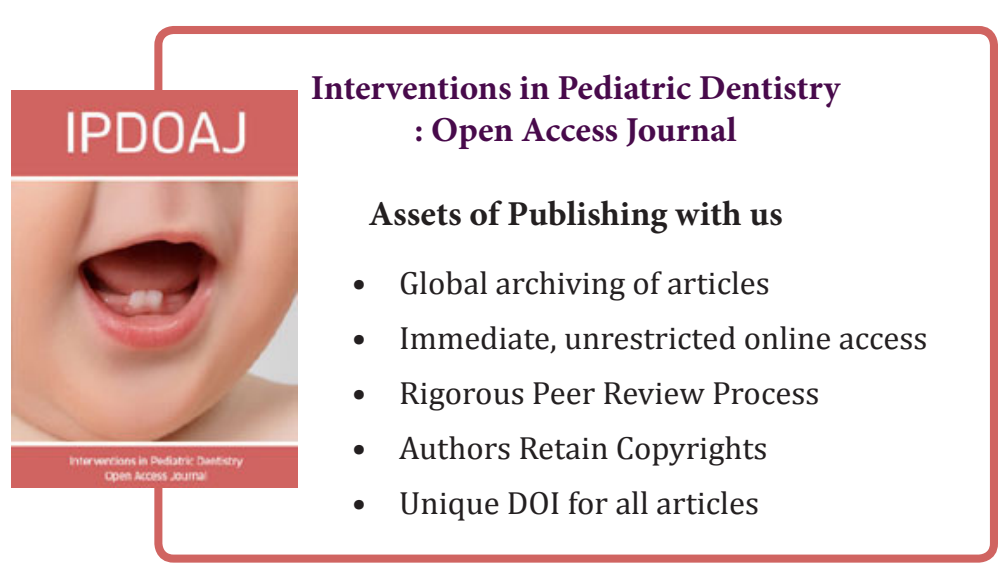

